\title{
Maternal small-bowel volvulus hindered by prodromal labor
}

\author{
Atsushi Imai • Hiroshi Toyoki • Tatsuro Furui • \\ Narutoshi Nagao
}

Received: 22 April 2007 / Accepted: 4 July 2007 /Published online: 16 August 2007

(C) Springer-Verlag 2007

\begin{abstract}
Small-bowel volvulus is a rare but accounts for $25 \%$ of the intestinal obstruction in pregnant women. Pregnancy and labor mask early signs and symptoms, leading to delay in diagnosis and increased morbidity. This report describes a case with bowel volvulus masked by early labor that underwent $330-\mathrm{cm}$ resection of small intestine and gave a live infant.
\end{abstract}

Keywords Pregnancy-complicating bowel volvulus . Intestinal infarction · Early labor

\section{Introduction}

Intestinal obstruction is a rare but serious complication of pregnancy, with significant maternal and fetal mortality; the incidence ranges from $1: 1,500$ to $1: 66,000$ deliveries [1-3] Small-bowel volvulus accounts for $25 \%$ of the intestinal obstruction in pregnant women but only $3-5 \%$ in nonpregnant women. Symptoms, including abdominal cramps and vomiting, are usually attributed to the normal discomforts of pregnancy and labor, resulting in delayed diagnosis $[4,5]$. Here we report a case of ileal volvulus that occurred at term treated with wide bowel resection.

\footnotetext{
A. Imai $(\bowtie) \cdot$ H. Toyoki $\cdot$ T. Furui

Department of Obstetrics and Gynecology,

Gifu University School of Medicine,

Yanagido, Gifu 501-1194, Japan

e-mail: atsushi@gifu-u.ac.jp

N. Nagao

Oncological Surgery, Gifu University School of Medicine,

Gifu, Japan
}

\section{Case report}

A 34-year-old woman at 37 weeks' and 2 days' gestation was admitted to the emergency department with sudden onset of abdominal and low back pains. All the routine antenatal tests by a local physician were in normal range. Her past history was not significant. She had had no abdominal operation. On admission, her vital signs included a temperature of $37.0 \mathrm{C}$, pulse of $110-120$ beats per minutes, and blood pressure of $85 / 60 \mathrm{mmHg}$. She had abdominal distention and vomiting with no bowel sounds. Fetal heart rate monitoring revealed a reactive pattern, and uterine contractions were slight. Abdominal computed tomography (CT) reveled a gas-fluid level in the distended small bowel and thickened wall, suggesting mechanical bowel infarction (Fig. 1a). The patient underwent an emergency, lowtransverse cesarean under general anesthesia through a vertical skin incision. She delivered a viable infant $(2,582 \mathrm{~g})$, with Apgar scores of 8 and 9 at 1 and $5 \mathrm{~min}$, respectively.

A large amount of ascites had been noted upon entering the peritoneal cavity, and subsequent examination of the abdominal cavity revealed a large amount of dusky, infarcted, and necrotic small bowel (Fig. 1b). A complete volvulus of dilated small bowel about its mesentery was identified. After detorsion, the gangrenous segment was covered with warm packing, and the team waited for reoxygenation. However, it was decided to proceed to a resection of all gangrenous midgut segment $(330 \mathrm{~cm})$, starting from jejunum, $120 \mathrm{~cm}$ after the ligament of Treiz, including the ileum up to $110 \mathrm{~cm}$ before the ileocecum. Jejunoileostomy was performed. She had total parenteral feeding until the 14th postoperative day and started oral feeding thereafter. 
Fig. 1 A case with small-bowel volvulus. a Computed tomography (transverse) section of the upper abdomen. The gas shadow (white arrow) and thickened and necrotic wall (black arrow) can be seen within the distended bowel. b Gangrenous bowel at operation after abdominal delivery
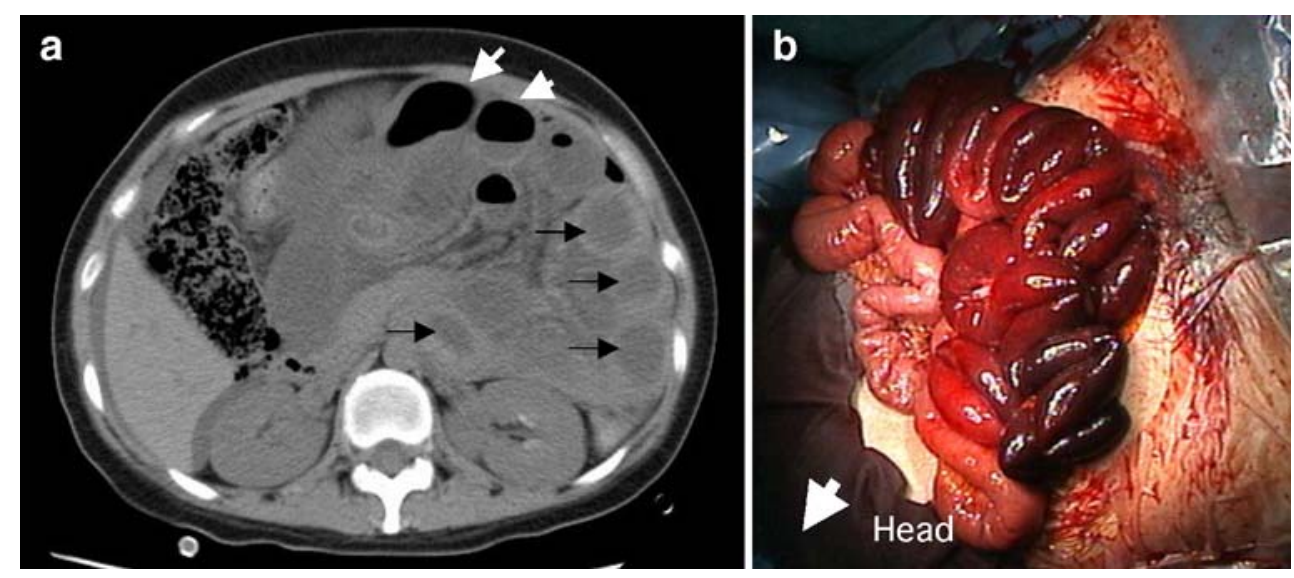

\section{Discussion}

The incidence of volvulus has been shown to increase with increasing gestation [3]. Obstruction occurs most frequently at times of rapid increases in uterine size (between 16 and 20 weeks and 32 and 36 weeks). In some cases, such as ours, there is an increase in occurrence at term because of a contraction-like change in uterine size. The intestinal volvulus resulting in closed-loop obstruction may cause vascular compromise acutely, thus necessitating emergency surgical intervention. Abdominal distension and pain are the symptoms usually seen in patients who have intestinal obstruction in pregnancy. These symptoms appear whatever the etiologic cause is. Pregnancy and labor mask early signs and symptoms, leading to delay in diagnosis and increased morbidity [3]. In our patient, 37 weeks' gestation might have superimposed on labor onset and contributed to intestinal compromise. In retrospect, our patient presented with an early sign of small bowel obstruction and irregular cramping, which was believed consistent with prodromal labor. She had no history of emesis, fever, or localized abdominal pain, which usually are seen with infracted bowel. Surgical evaluation must be used liberally because medical and conservative treatment methods have less effect in pregnants than in normal population [1]. As in our case, partial resection may be needed. Cesarean section is the choice for giving birth, as it gives a chance for the surgical evaluation of obstruction. The delay from presentation to definitive management continues to result in significant morbidity and mortality.

\section{References}

1. Baykal C, Al A, Ozer S, Hizli D, Gokcin H (2006) Ileal resection for gangrenous ileal volvulus in a term pregnancy: A case report. Arch Gynecol Obstet 273:304-306

2. Dilbaz S, Gelisen O, Caliskan E, Caliskan S, Gokcin H, Haberal A (2003) Small bowel volvulus in pregnancy. Eur J Obstet Gynecol Reprod Biol 111:204-206

3. Ventura-Braswell A, Satin A, Higby K (1998) Delayed diagnosis of bowel infarction secondary to maternal midgut volvulus at term. Obstet Gynecol 91:808-810

4. Singla S, Kadian Y, Goyal A, Sharma U, Kadian N (2005) Caecal volvulus in pregnancy: Is delay in diagnosis avoidable? Asian J Surg 28:52-54

5. Huang J, Shin J, Huang Y, Chao C, Ho S, Wu M, Huang T, Chang F, Ying K, Chang L (2005) Small bowel volvulus among adults. J Gastroenterol Hepatol 20:1906-1912 